Claremont Colleges

Scholarship@ Claremont

All HMC Faculty Publications and Research

HMC Faculty Scholarship

$1-1-1997$

\title{
Positive Solution Curves of Semipositone Problems with Concave Nonlinearities
}

\author{
Alfonso Castro \\ Harvey Mudd College \\ Sudhasree Gadam \\ University of North Texas \\ Ratnasingham Shivaji \\ Mississippi State University
}

\section{Recommended Citation}

A. Castro, S. Gadam and R. Shivaji. “Positive solutions curves of semipositone problems with concave nonlinearities”, Proc. Royal Society of Edinburgh, Vol. 127A (1997), pp. 921-934.

This Article is brought to you for free and open access by the HMC Faculty Scholarship at Scholarship @ Claremont. It has been accepted for inclusion in All HMC Faculty Publications and Research by an authorized administrator of Scholarship @ Claremont. For more information, please contact scholarship@cuc.claremont.edu. 


\title{
Positive solution curves of semipositone problems with concave nonlinearities*
}

\author{
Alfonso Castro \\ Department of Mathematics, University of North Texas, Denton, \\ TX 76203-5116, U.S.A. \\ Sudhasree Gadam \\ Mathematics, Yashodha, J.C.R. VI Cross, Chitradurga, India 577501 \\ R. Shivaji \\ Department of Mathematics, Mississippi State University, \\ Mississippi State, MS 39762, U.S.A.
}

(MS received 11 June 1996)

We consider the positive solutions to the semilinear equation:

$$
\begin{gathered}
-\Delta u(x)=\lambda f(u(x)) \text { for } x \in \Omega, \\
u(x)=0 \text { for } x \in \partial \Omega,
\end{gathered}
$$

where $\Omega$ denotes a smooth bounded region in $\mathbb{R}^{N}(N>1)$ and $\lambda>0$. Here $f:[0, \infty) \rightarrow \mathbb{R}$ is assumed to be monotonically increasing, concave and such that $f(0)<0$ (semipositone). Assuming that $f^{\prime}(\infty) \equiv \lim _{t \rightarrow \infty} f^{\prime}(t)>0$, we establish the stability and uniqueness of large positive solutions in terms of $(f(t) / t)^{\prime}$. When $\Omega$ is a ball, we determine the exact number of positive solutions for each $\hat{\lambda}>0$. We also obtain the geometry of the branches of positive solutions completely and establish how they evolve. This work extends and complements that of $[3,7]$ where $f^{\prime}(\infty) \leqq 0$.

\section{Introduction}

We consider the positive solutions to the semilinear equation:

$$
\begin{gathered}
-\Delta u(x)=\lambda f(u(x)) \text { for } x \in \Omega, \\
u(x)=0 \text { for } x \in \partial \Omega,
\end{gathered}
$$

where $\Omega$ denotes a smooth bounded region in $\mathbb{R}^{N}(N>1)$ and $\lambda>0$. Here $f:[0, \infty) \rightarrow \mathbb{R}$ is assumed to be monotonically increasing, concave and such that

$$
f(0)<0 \text { (semipositone), } f(t)>0 \text { for some } t>0 .
$$

We define $F$ by $F(t)=\int_{0}^{t} f(s) d s$ and let $\beta$ and $\theta$ denote the unique positive zeros of $f$ and $F$, respectively.

\footnotetext{
* This research was partially supported by NSF grant DMS 9215027.
} 
It is easy to show that either

$$
(f(t) / t)^{\prime}>0 \text { for all } t>0,
$$

or

there exists an $\eta>0 \quad$ such that $\eta f^{\prime}(\eta)=f(\eta) ; \quad(f(t) / t)^{\prime}>0$

$$
\text { for all } t \in(0, \eta) \text { and }(f(t) / t)^{\prime}<0 \text { for all } t \in(\eta, \infty) \text {, }
$$

and, moreover, that $f^{\prime}(\infty):=\lim _{t \rightarrow \infty} f^{\prime}(t)>0$ in the case where (1.4) holds. Therefore if $f^{\prime}(\infty)=0$, then $f$ must satisfy (1.5). Moreover, (1.4)-(1.5) are determined by whether or not $h(t) \equiv f(t)-t f^{\prime}(\infty)$ is negative. More precisely, we have:

Remark 1.1. Condition (1.4) holds if and only if $h(t)<0$ for all $t \in[0, \infty)$. Conversely, (1.5) holds if and only if $h$ has a positive zero.

Here we consider the case where

$$
f^{\prime}(\infty)>0 .
$$

The case when $\Omega$ is a ball and $f^{\prime}(\infty)=0$ has been completely classified in [3] (see Theorem 1.4). Also, the case when $\Omega$ is a ball, $f$ is no longer monotone and $f^{\prime}(\infty) \leqq 0$ has been completely classified in [7] (see Remark 1.5). For results when $\Omega$ is a general domain and $f^{\prime}(\infty)=0$, see [6].

Notation. Let $\mu_{i}, i=1,2, \ldots$ denote the eigenvalues of

$$
\begin{gathered}
-\Delta \varphi(x)=\mu_{i} \varphi(x) \quad \text { for } x \in \Omega, \\
\varphi(x)=0 \text { for } x \in \partial \Omega .
\end{gathered}
$$

Our main results are:

THEOREM 1.2. If (1.6) holds, then:

(i) for $\lambda$ 's near $0,(1.1)-(1.2)$ has no positive solution;

(ii) if (1.4) holds, then positive solutions to (1.1)-(1.2) are unstable;

(iii) for $\lambda$ in bounded intervals, large positive solutions to (1.1)-(1.2) are unique. If (1.5) holds, such solutions are stable.

THEOREM 1.3. Let $\Omega$ denote the unit ball centred at the origin in $\mathbb{R}^{N}$. Assume that (1.6) holds. Then there exist $0<\lambda_{1}<\lambda_{2}<\infty$ such that:

(i) if (1.4) holds then $\lambda_{1}=\mu_{1} / f^{\prime}(\infty)$ and (1.1)-(1.2) has a positive solution if and only if $\lambda_{1}<\lambda \leqq \lambda_{2}$. Moroever, such a solution is unstable (see Fig. 1.1);

(ii) if (1.5) holds, then $\lambda_{1}<\mu_{1} / f^{\prime}(\infty)$ and for $\lambda=\lambda_{1}$ the problem (1.1)-(1.2) has exactly one positive solution; it is unstable. If $\mu_{1} / f^{\prime}(\infty)<\lambda_{2}$, then for $\lambda \in\left(\lambda_{1}, \mu_{1} / f^{\prime}(\infty)\right)$ the problem (1.1)-(1.2) has exactly one stable and one unstable positive solution. For $\lambda \in\left[\mu_{1} / f^{\prime}(\infty), \lambda_{2}\right]$, the above problem has exactly one positive solution and it is unstable (see Fig. 3.1). If $\mu_{1} / f^{\prime}(\infty) \geqq \lambda_{2}$, then for $\lambda \in\left(\lambda_{1}, \lambda_{2}\right]$ the problem (1.1)-(1.2) has exactly two positive solutions; one stable and one unstable. For $\lambda \in\left(\lambda_{2}, \mu_{1} / f^{\prime}(\infty)\right)$, the problem (1.1)-(1.2) has exactly one positive solution and it is stable (see Fig. 3.1).

The following theorem and remark are from $[3,7]$ and we include them here for the sake of completeness.

THEOREM 1.4. Assume that $f^{\prime}(\infty)=0$. Then there exist $0<\lambda_{1}<\lambda_{2}<\infty$ such that for 


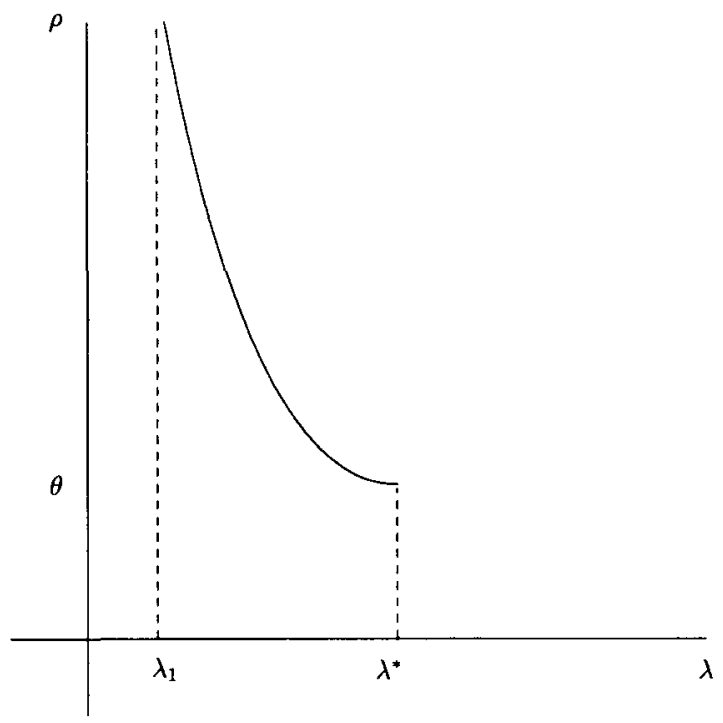

Figure 1.1. $f^{\prime}(\infty)>0$ and $(1.4)$ holds.
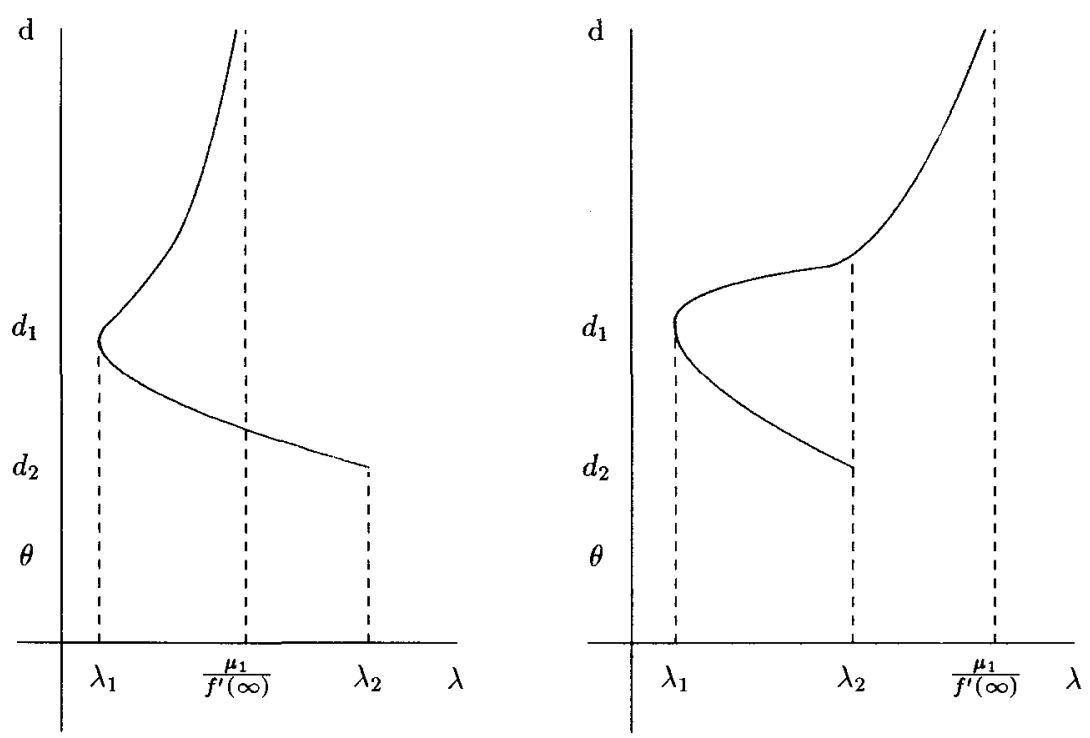

Figure 3.1. $f^{\prime}(\infty)>0$ and (1.5) holds.

$\lambda \in\left(\lambda_{1}, \lambda_{2}\right]$ the problem (1.1)-(1.2) has exactly two positive solutions, one stable and one unstable. For $\lambda=\lambda_{1}$, the problem (1.1)-(1.2) has exactly one positive solution and it is stable (see Fig. 3.2).

REMARK 1.5. Theorem 1.4 also holds if $f$ is assumed to be concave with $f(0)<0$ and $f(\gamma)=0$ for some $\gamma>\theta$ (see [7]).

REMARK 1.6. The author of [9], while studying classes of concave nonlinearities, in 


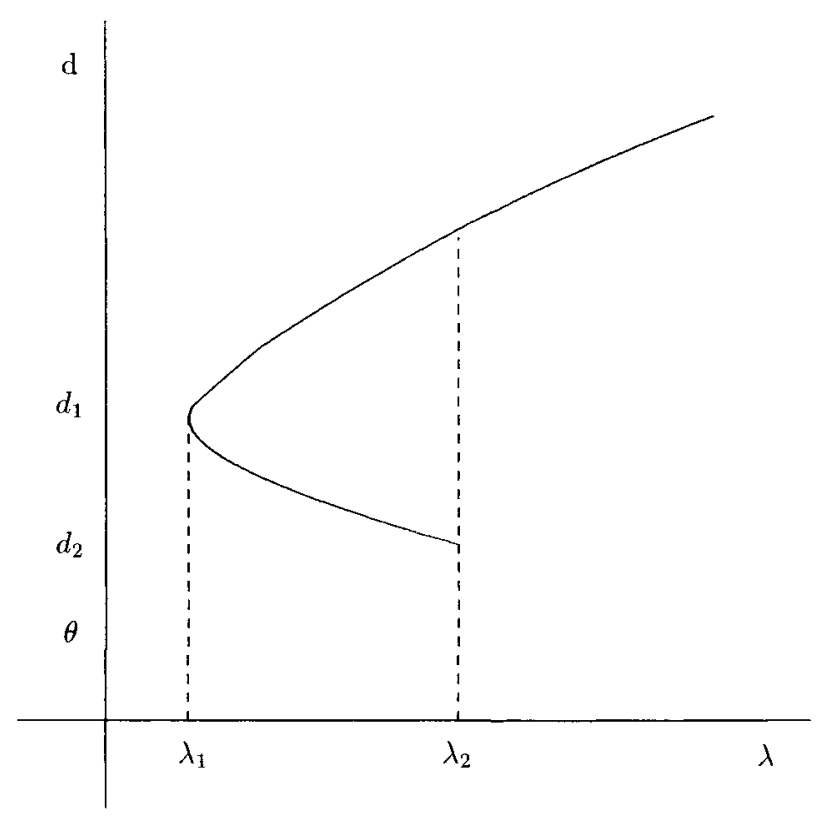

Figure 3.2. $f^{\prime}(\infty)=0$.

the case when $N=1$, discusses the possibility of situations where there exist $0<\lambda^{*}<\lambda^{* *}$ such that for $0<\lambda<\lambda^{*}$ there are no positive solutions and for $\lambda^{*} \leqq \lambda<\lambda^{* *}$ there is a unique positive solution. Our results here prove that this does not occur. For proofs of the corresponding results for the case $N=1$, see [5].

Our proofs use eigenvalue comparison arguments and bifurcation analysis. For other results about solutions of (1.1)-(1.2), the reader is referred to $[2,7]$. In order to prove Theorem 1.2, we use the fact that (1.1)-(1.2) has large positive solutions in arbitrary bounded regions $\Omega$ in $\mathbb{R}^{N}$. Our methods use the properties derived from bifurcation from infinity (see [2]).

\section{Evolution of positive solution curves}

From Theorems 1.2, 1.3 and 1.4, we now can deduce the evolution of the bifurcation curves as the nonlinearity changes from satisfying (1.4) to satisfying (1.5). For example, consider $f(s, t)=(1-s) f_{1}(t)+s f_{2}(t)$ with $f_{1}$ satisfying $(1.4)$ and $f_{2}$ satisfying (1.5). If $f_{2}^{\prime}(\infty)>0$, then the positive solution curves evolve from Figure 1.1 to Figure 3.1 as $s$ varies from 0 to 1 . Moreover, if $f_{2}^{\prime}(\infty)=0$, then the curves evolve from Figure 1.1 to Figure 3.2, passing through Figure 3.1.

\section{Existence and stability of positive solutions in general regions}

First we note that if $f$ satisfies (1.6), then, by [2, Theorem 1], the problem (1.1)-(1.2) has positive solutions $(\lambda, u)$ near $\left(\mu_{1} / f^{\prime}(\infty), \infty\right)$. Let $\varphi_{1}>0$ be the eigenfunction corresponding to $\mu_{1}$ in (1.7) with $\left\|\varphi_{1}\right\|_{\infty}=1$. Since large solutions to (1.1)-(1.2) 
bifurcate from $\left(\mu_{1} / f^{\prime}(\infty), \infty\right)$ and $\mu_{1} / f^{\prime}(\infty)$ is a simple eigenvalue, by the results of [8] we see that there exists an $\varepsilon_{*}>0, K_{*}>0$ and $d_{*}>0$ and continuous functions $\Lambda:\left[K_{*}, \infty\right) \rightarrow \mathbb{R}$ and $w:\left[K_{*}, \infty\right) \rightarrow\left\{u \in C^{1}(\Omega): \int_{\Omega} u \varphi_{1}=0\right\}$ such that if $\|u\|_{\infty}>d_{*}$, $u>0$ and $\left|\lambda-\mu_{1} / f^{\prime}(\infty)\right|<\varepsilon_{*}$ with $(\lambda, u)$ is a solution to (1.1)-(1.2), then

$$
u=K \varphi_{1}+w(K), \quad \lambda=\Lambda(K),
$$

for some $K \in\left[K_{*}, \infty\right)$. Also, $\Lambda(K) \rightarrow \mu_{1} / f^{\prime}(\infty)$ as $K \rightarrow \infty$ and $\|w\|_{c^{1}}=o(K)$. That is, $\|u\|_{\infty} / K=O(1)$. Note that $\partial \varphi_{1} / \partial v>0$ on $\partial \Omega$, where $v$ denotes the inward unit normal vector. By the compactness of $\partial \Omega$, there exists a $C_{1}>0$ such that

$$
\varphi_{1}(x) \geqq C_{1} \operatorname{dist}(x, \partial \Omega),
$$

for all $x \in \Omega$. On the other hand, since $\|w(K)\|_{C^{1}}=o(K)$ as $K \rightarrow \infty$, there exists a $K_{0} \geqq K_{*}$ such that

$$
|w(x)| \leqq\left(C_{1} / 2\right) \operatorname{dist}(x, \partial \Omega) K,
$$

for $K>K_{0}$. So, for $K>K_{0}$, we obtain

$$
u(x) \geqq\left(C_{1} / 2\right) \operatorname{dist}(x, \partial \Omega) K,
$$

for all $x \in \Omega$.

Remark 3.1. From (3.3), it follows that $\|u\|_{\infty} \rightarrow \infty$ uniformly on compact subsets of $\Omega$ as $K \rightarrow \infty$.

In the following lemmas, we determine the stability and the uniqueness of the positive solutions to (1.1)-(1.2) as a function of (1.4) and (1.5).

Lemma 3.2. If (1.6) holds, then there exists $\varepsilon^{*} \in\left(0, \varepsilon_{*}\right)$ and $K^{*}>K_{*}$ such that if $\left|\lambda-\mu_{1} / f^{\prime}(\infty)\right|<\varepsilon^{*}$, then (1.1)-(1.2) has at most one positive solution with $\|u\|_{\infty}>K^{*}$. In particular, the function $\Lambda$ in (3.1) is one-to-one on $\left[K^{*}, \infty\right)$.

Proof. Let $u_{1}=K_{1} \varphi_{1}+w\left(K_{1}\right)$ and $u_{2}=K_{2} \varphi_{1}+w\left(K_{2}\right)$ be two positive solutions to (1.1)-(1.2) with $K_{1}, K_{2}$ large and for some $\lambda$ close to $\mu_{1} / f^{\prime}(\infty)$. Without loss of generality, we may assume that $K_{1}>K_{2}$. We write $w_{i}=w\left(K_{i}\right), i=1,2$. From (1.1), we have

$$
-\Delta\left(u_{1}-u_{2}\right)=\lambda\left(f\left(u_{1}\right)-f\left(u_{2}\right)\right) .
$$

Multiplying (3.4) by $w_{1}-w_{2}$ and integrating by parts, we have

$$
\int_{\Omega}\left|\nabla\left(w_{1}-w_{2}\right)\right|^{2}=\lambda \int_{\Omega}\left[f^{\prime}(\infty)\left(w_{1}-w_{2}\right)+\left(h\left(u_{1}\right)-h\left(u_{2}\right)\right)\right]\left(w_{1}-w_{2}\right),
$$

where $h(t) \equiv f(t)-t f^{\prime}(\infty)$ (see Remark 1.1). From (3.3), we have

$$
u_{i}(x) \geqq\left(C_{1} / 2\right) K_{i} \operatorname{dist}(x, \partial \Omega),
$$

for all $x \in \Omega$. By the Sobolev Embedding Theorem (see [1]), there exists a positive constant $C(\Omega) \equiv C_{2}$ such that

$$
\left(\int_{\Omega} y^{2 N /(N-2)}\right)^{(N-2) / 2 N} \leqq C_{2}\left(\int_{\Omega}|\nabla y|^{2}\right)^{\frac{1}{2}} \text { for any } y \in H_{0}^{1}(\Omega) .
$$


Also, by the variational characterisation of eigenvalues to $-\Delta$, we know that

$$
\int_{\Omega}|\nabla y|^{2} \geqq \mu_{2} \int_{\Omega} y^{2},
$$

for any $y \in H_{0}^{1}(\Omega)$ with $y$ orthogonal to $\varphi_{1}$. Let $\varepsilon>0$ be such that

$$
3 \varepsilon \lambda<\mu_{2}-\mu_{1} \text { for }\left|\lambda-\frac{\mu_{1}}{f^{\prime}(\infty)}\right|<\varepsilon_{*} .
$$

Later we will restrict $\varepsilon$ further. Let $M=M(\varepsilon)$ be such that if $t \geqq M$ then $h^{\prime}(t)<\varepsilon$. By (3.3), there exists $C_{3}>0$ such that, for $K$ sufficiently large,

$$
|\{x \in \Omega: u(x) \leqq M\}| \leqq C_{3} / K .
$$

Here $|\cdot|$ denotes the Lebesgue measure. Let $\varepsilon^{*}>0$ and $K^{*}>K_{*}$ be such that if

$$
\left|\lambda-\frac{\mu_{1}}{f^{\prime}(\infty)}\right|<\varepsilon^{*}
$$

then

$$
\frac{\left|\lambda f^{\prime}(\infty)-\mu_{1}\right|}{\mu_{2}}+h^{\prime}(0) C_{2}^{2}\left(2 C_{3} / K^{*}\right)^{4 / N}<\frac{\mu_{2}-\mu_{1}}{3} .
$$

Let $\Omega_{1}=\left\{x \in \Omega: u_{1}(x) \leqq M\right.$, or $\left.u_{2} \geqq M\right\}$ and $\Omega_{2}=\Omega \backslash \Omega_{1}$. Thus, by the Mean Value Theorem,

$$
\begin{aligned}
\int_{\Omega_{1}} & \left(h\left(u_{1}\right)-h\left(u_{2}\right)\right)\left(w_{1}-w_{2}\right) \\
= & \int_{\Omega_{1}} h^{\prime}(\zeta)\left(u_{1}-u_{2}\right)\left(w_{1}-w_{2}\right) \\
\leqq & \int_{\Omega_{1}} h^{\prime}(0)\left|u_{1}-u_{2}\right|\left|w_{1}-w_{2}\right| \\
\leqq & h^{\prime}(0)\left\|w_{1}-w_{2}\right\|_{L^{2}\left(\Omega_{1}\right)}\left(\left(K_{1}-K_{2}\right)\left|\Omega_{1}\right|^{\frac{1}{2}}+\left\|w_{1}-w_{2}\right\|_{L^{2}\left(\Omega_{1}\right)}\right) \\
\leqq & h^{\prime}(0)\left\|w_{1}-w_{2}\right\|_{L^{2 N / N-2)}\left(\Omega_{1}\right)}\left|\Omega_{1}\right|^{2 / N} \\
& \times\left(\left(K_{1}-K_{2}\right)|\Omega|^{\frac{1}{2}}+\left\|w_{1}-w_{2}\right\|_{L^{2 N /(N-2)}\left(\Omega_{1}\right)}\left|\Omega_{1}\right|^{2 / N}\right) .
\end{aligned}
$$

On the other hand, by (3.9) for $K_{1}, K_{2}$ sufficiently large, we have

$$
\left|\Omega_{1}\right| \leqq 2 C_{3}\left(1 / K_{2}\right) \text {. }
$$

Also,

$$
\begin{aligned}
& \int_{\Omega_{2}}\left(h\left(u_{1}\right)-h\left(u_{2}\right)\right)\left(w_{1}-w_{2}\right) \\
& \quad \leqq \int_{\Omega_{2}} \varepsilon\left|u_{1}-u_{2}\right|\left|w_{1}-w_{2}\right| \\
& \quad \leqq \varepsilon\left\|w_{1}-w_{2}\right\|_{L^{2}}\left(\left(K_{1}-K_{2}\right)|\Omega|^{\frac{1}{2}}+\left\|w_{1}-w_{2}\right\|_{L^{2}}\right)
\end{aligned}
$$




$$
\leqq \frac{\varepsilon}{\sqrt{\mu_{2}}}\left\|w_{1}-w_{2}\right\|_{H^{1}}\left(\left(K_{1}-K_{2}\right)|\Omega|^{\frac{1}{2}}+\left(1 / \sqrt{\mu_{2}}\right)\left\|w_{1}-w_{2}\right\|_{H^{1}}\right)
$$

in view of (3.8). From (3.5), (3.7) and (3.11)-(3.13), we obtain

$$
\begin{aligned}
& \left(1-\frac{\lambda f^{\prime}(\infty)}{\mu_{2}}-\lambda h^{\prime}(0) C_{2}^{2}\left|\Omega_{1}\right|^{4 / N}-\frac{\lambda \varepsilon}{\mu_{2}}\right)\left\|w_{1}-w_{2}\right\|_{H^{1}} \\
\leqq & \frac{\varepsilon}{\sqrt{\mu_{2}}}\left|K_{1}-K_{2}\right||\Omega|^{\frac{1}{2}}+C_{2} \lambda h^{\prime}(0)\left|K_{1}-K_{2}\right||\Omega|^{\frac{1}{2}}\left|\Omega_{1}\right|^{2 / N} .
\end{aligned}
$$

By (3.7), (3.10) and the assumption that $3 \varepsilon \lambda<\mu_{2}-\mu_{1}$, we conclude that

$$
\left\|w_{1}-w_{2}\right\|_{L^{2 N /(N-2)}} \leqq O\left(\left(1 / K_{2}\right)^{2 / N}+\varepsilon\right)\left(K_{1}-K_{2}\right),
$$

as $K_{1} \rightarrow \infty$ and $K_{2} \rightarrow \infty$. Let $P: L^{2}(\Omega) \rightarrow L^{2}(\Omega)$ denote the orthogonal projection onto $\left\{u \in L^{2}(\Omega): \int_{\Omega} u \varphi_{1}=0\right\}$. Then from (1.1) we have

$$
-\Delta\left(w_{1}-w_{2}\right)=\lambda f^{\prime}(\infty)\left(w_{1}-w_{2}\right)+P\left(h\left(u_{1}\right)-h\left(u_{2}\right)\right) .
$$

Multiplying (3.4) by $\varphi_{1}$ and integrating by parts, we obtain that

$$
\int_{\Omega}\left(h\left(u_{1}\right)-h\left(u_{2}\right)\right) \varphi_{1}=O\left(e^{*}\right)\left(K_{1}-K_{2}\right) .
$$

Consider

$$
\begin{aligned}
& \left(\int_{\Omega}\left[\left(h\left(u_{1}\right)-h\left(u_{2}\right)\right)\right]^{2 N /(N-2)}\right)^{(N-2) / 2 N} \\
& \leqq\left(\int_{\Omega_{1}}\left[\left(h\left(u_{1}\right)-h\left(u_{2}\right)\right)\right]^{2 N /(N-2)}+\int_{\Omega_{2}}\left[\left(h\left(u_{1}\right)-h\left(u_{2}\right)\right)\right]^{2 N /(N-2)}\right)^{(N-2) / 2 N} \\
& \leqq h^{\prime}(0)\left(\int_{\Omega_{1}}\left[\left(K_{1}-K_{2}\right) \varphi_{1}+\left|w_{1}-w_{2}\right|\right]^{2 N /(N-2)}\right)^{(N-2) / 2 N} \\
& +\varepsilon\left(\int_{\Omega_{2}}\left[\left(K_{1}-K_{2}\right) \varphi_{1}+\left|w_{1}-w_{2}\right|\right]^{2 N /(N-2)}\right)^{(N-2) / 2 N} \\
& \leqq\left(h^{\prime}(0)\left|\Omega_{1}\right|^{(N-2) / 2 N}+\varepsilon|\Omega|^{(N-2) / 2 N}\right)\left(K_{1}-K_{2}\right)+\left(h^{\prime}(0)+\varepsilon\right)\left\|w_{1}-w_{2}\right\|_{L^{2 N / N-}-(23.18)}
\end{aligned}
$$

From (3.15)-(3.18) and by a priori estimates for solutions to elliptic boundary value problems, we obtain

$$
\left\|w_{1}-w_{2}\right\|_{H^{2,2 N / N-2)}}=\left(K_{1}-K_{2}\right) O\left(\left(1 / K_{2}\right)^{2 / N}+\varepsilon+\left(1 / K_{2}\right)^{(N-2) / 2 N}\right) .
$$

Now, using a boot-strap argument and the Sobolev Embedding Theorem, this gives

$$
\left\|w_{1}-w_{2}\right\|_{C^{1}(\Omega)}=\left(K_{1}-K_{2}\right) O\left(\left(1 / K_{2}\right)^{2 / N}+\varepsilon+\left(1 / K_{2}\right)^{(N-2) / 2 N}\right) .
$$

Thus there exists an $\varepsilon^{*}$ and $K^{*}$ such that if, for some $\lambda$ with $\left|\lambda-\mu_{1} / f^{\prime}(\infty)\right|<\varepsilon^{*}$, we have $u_{1}=K_{1} \varphi_{1}+w\left(K_{1}\right)$ and $u_{2}=K_{2} \varphi_{1}+w\left(K_{2}\right)$ as two positive solutions to (1.1)-(1.2) with $K_{1}>K_{2}>K^{*}$, then $u_{1}(x)>u_{2}(x)$ for all $x \in \Omega$ (see (3.3)). Now, we use the concavity of $f$ to arrive at a contradiction. From (3.4) and the Mean Value 
928

A. Castro et al.

Theorem, we get

$$
-\Delta\left(u_{1}-u_{2}\right)=\lambda f^{\prime}(\zeta)\left(u_{1}-u_{2}\right),
$$

where $u_{1} \geqq \zeta \geqq u_{2}$. Multiplying this by $u_{1}$ and integrating by parts, we obtain

$$
0=\int_{\Omega}\left(f\left(u_{1}\right)-u_{1} f^{\prime}(\zeta)\right)\left(u_{1}-u_{2}\right) \leqq \int_{\Omega}\left(f\left(u_{1}\right)-u_{1} f^{\prime}\left(u_{1}\right)\right)\left(u_{1}-u_{2}\right)
$$

using the fact that $f$ is concave. If (1.4) holds, (3.21) shows that $u_{1}=u_{2}$. On the other hand, if $f$ satisfies (1.5), we let $B_{1}>0$ be such that, for all $t \geqq \eta+1$,

$$
f(t)-t f^{\prime}(t) \geqq B_{1} \text {. }
$$

From (3.9), if $A:=\left\{x: u_{2}(x) \leqq \eta+1\right\}$, then $\mu(A)=O\left(1 / K_{2}\right)$. Also, from (3.19) we have $u_{1}(x)-u_{2}(x) \geqq\left(K_{1}-K_{2}\right)$ dist $(x, \partial \Omega) O(1)$. Hence $\int_{\Omega} u_{1}-u_{2} \geqq\left(K_{1}-K_{2}\right) O(1)$. Thus for $K^{*}$ sufficiently large,

$$
\begin{aligned}
\int_{\Omega} & {\left[f\left(u_{2}\right)-f^{\prime}\left(u_{2}\right) u_{2}\right]\left(u_{1}-u_{2}\right) } \\
& =\int_{\Omega \backslash A}\left[f\left(u_{2}\right)-f^{\prime}\left(u_{2}\right) u_{2}\right]\left(u_{1}-u_{2}\right)+\int_{A}\left[f\left(u_{2}\right)-f^{\prime}\left(u_{2}\right) u_{2}\right]\left(u_{1}-u_{2}\right) \\
& \geqq B_{1} \int_{\Omega \backslash A}\left(u_{1}-u_{2}\right)-\left(K_{1}-K_{2}\right) O\left(1 / K_{2}^{2}\right) \\
& =B_{1} \int_{\Omega}\left(u_{1}-u_{2}\right)-\left(K_{1}-K_{2}\right) O\left(1 / K_{2}^{2}\right) \geqq\left(K_{1}-K_{2}\right) O(1),
\end{aligned}
$$

where we have used that $\int_{A}\left(u_{1}-u_{2}\right) \leqq\left(K_{1}-K_{2}\right) o(1)$. Also, multiplying (3.20) by $u_{2}$ and integrating by parts, we get

$$
0=\int_{\Omega}\left[f\left(u_{2}\right)-u_{2} f^{\prime}(\zeta)\right]\left(u_{1}-u_{2}\right) \geqq \int_{\Omega}\left[f\left(u_{2}\right)-u_{2} f^{\prime}\left(u_{2}\right)\right]\left(u_{1}-u_{2}\right),
$$

which contradicts (3.23). Thus the lemma is proved.

LEMMA 3.3. If (1.4) holds, then any positive solution to (1.1)-(1.2) is unstable. Moreover, there exist a continuous decreasing function $d:\left(\mu_{1} / f^{\prime}(\infty)\right.$, $\left(\mu_{1} / f^{\prime}(\infty)+\varepsilon^{*}\right) \rightarrow\left(K^{*},+\infty\right)$ such that $\lim _{\lambda \rightarrow \mu_{1} / f^{\prime}(\infty)} d(\hat{\lambda})=\infty$. If $\|u\|_{\infty} \geqq K^{*}$ and $\left|\lambda-\mu_{1} / f^{\prime}(\infty)\right|<\varepsilon^{*}$, then $u=u(\cdot, \lambda, d(\lambda))$. That is, large positive solutions to (1.1)-(1.2) are unique for $\lambda$ near $\mu_{1} / f^{\prime}(\infty)$.

Proof. Let $u$ be a positive solution to (1.1)-(1.2) and let $\rho_{i}, i=1,2, \ldots$ denote eigenvalues of

$$
\begin{gathered}
-\Delta \psi(x)=\lambda f^{\prime}(u(x)) \psi(x)+\rho \psi(x) \text { for } x \in \Omega, \\
\psi(x)=0 \text { for } x \in \partial \Omega .
\end{gathered}
$$

Let $\psi_{1}$ be an eigenfunction corresponding to the smallest eigenvalue $\rho_{1}$ and chosen to be positive in $\Omega$. Now multiplying (1.1) by $\psi_{1}$ and (3.25) by $u$, subtracting one 
from the other and integrating over $\Omega$, we obtain

$$
\lambda \int_{\Omega}\left[f^{\prime}(u(x)) u(x)-f(u(x))\right] \psi_{1}(x) d x=-\rho_{1} \int_{\Omega} \psi_{1}(x) u(x) d x .
$$

In view of (1.4), we conclude that $\rho_{1}<0$ and hence from the theory of linearised stability $u$ is unstable. Finally, from the results of [2] and Lemma 3.2, the existence of the function $d$ follows.

Lemma 3.4. If (1.5) holds, then large positive solutions to (1.1)-(1.2) are stable. Moreover, there exists a continuous increasing function $d:\left(\left(\mu_{1} / f^{\prime}(\infty)-\varepsilon^{*}\right.\right.$, $\mu_{1} / f^{\prime}(\infty) \rightarrow\left(K^{*},+\infty\right)$ such that $\lim _{\lambda \rightarrow \mu_{1} / f^{\prime}(\infty)} d(\lambda)=\infty$. If $\|u\|_{\infty} \geqq K^{*}$ and $\left|\lambda-\mu_{1} / f^{\prime}(\infty)\right|<\varepsilon^{*}$, then $u=u(\cdot, \lambda, d(\lambda))$. That is, large positive solutions to $(1.1)-(1.2)$ are unique for $\lambda$ near $\mu_{1} / f^{\prime}(\infty)$.

Proof. Let $\rho_{1}$ and $\psi_{1}>0$ be as in (3.25)-(3.26). Suppose, on the contrary, that $\rho_{1} \leqq 0$. Without loss of generality, we may assume that $\int_{\Omega} \psi_{1}=1$. Thus we have

$$
\int_{\Omega}\left|\nabla \psi_{1}\right|^{2}=\lambda \int_{\Omega} f^{\prime}(u(x)) \psi_{1}^{2}+\rho_{1} \int_{\Omega} \psi_{1}^{2} \leqq \lambda f^{\prime}(0) \int_{\Omega} \psi_{1}^{2}
$$

Since $\psi_{1} \in H_{0}^{1}(\Omega)$, from (3.11) we have

$$
\left(\int_{\Omega} \psi_{1}^{2 N(N-2)}\right)^{(N-2) / 2 N} \leqq C\left(\int_{\Omega}\left|\nabla \psi_{1}\right|^{2}\right)^{\frac{1}{2}}
$$

Since $\psi_{1} \in L^{2 N /(N-2)}$, by the generalised Hölder inequality we have

$$
\left(\int_{\Omega} \psi_{1}^{2}\right)^{\frac{1}{2}} \leqq\left(\int_{\Omega} \psi_{1}^{2 N /(N-2)}\right)^{a((N-2) / 2 N)}\left(\int_{\Omega} \psi_{1}\right)^{1-a},
$$

where $a=N /(N+2)$. Now, from (3.27), (3.28) and (3.29), we obtain

$$
\begin{aligned}
\left(\int_{\Omega} \psi_{1}^{2 N /(N-2)}\right)^{(N-2) / 2 N} & \leqq C\left(\lambda f^{\prime}(0)\right)^{\frac{1}{2}}\left(\int_{\Omega} \psi_{1}^{2}\right)^{\frac{1}{2}} \\
& \leqq C\left(\lambda f^{\prime}(0)\right)^{\frac{1}{2}}\left(\int_{\Omega} \psi_{1}^{2 N /(N-2)}\right)^{a((N-2) / 2 N)},
\end{aligned}
$$

using the assumption that $\int_{\Omega} \psi_{1}=1$. Thus we obtain $B=B(\lambda, \Omega, f)$, with

$$
\left\|\psi_{1}\right\|_{L^{2 N /(N-2)} \leqq B}
$$

Now, using (1.1) and (3.25), we obtain

$$
\lambda \int_{\Omega}\left[f(u(x))-f^{\prime}(u(x)) u(x)\right] \psi_{1}(x) d x=\rho_{1} \int_{\Omega} \psi_{1}(x) u(x) d x .
$$

Let $\eta_{1}>\eta$. Since $(f(t) / t)^{\prime}<0$ for $t>\eta$ and $f$ is concave, there exists $B_{1}>0$ such that, for all $t \geqq \eta_{1}$, we have

$$
f(t)-t f^{\prime}(t) \geqq B_{1} .
$$

From the bifurcation properties we have, if $A:=\left\{x: u(x) \leqq \eta_{1}\right\}$, then $\mu(A)=$ 
$O\left(1 /\|u\|_{\infty}\right)$ as $\|u\|_{\infty} \rightarrow \infty(\operatorname{see}(3.4))$. Thus

$$
\begin{aligned}
\int_{A}\left[f(u)-f^{\prime}(u) u\right] \psi_{1} & \leqq K \int_{A} \psi_{1} \\
& \leqq\left(\int_{\Omega} \psi_{1}^{2 N /(N-2)}\right)^{a((N-2) / 2 N)}\left(\int_{\Omega} \psi_{1}\right)^{1-a}
\end{aligned}
$$

and hence $\rightarrow 0$ as $\|u\| \rightarrow \infty$ (using the boundedness in (3.30)). Now, in view of (3.32) and (3.33), we get

$$
\begin{aligned}
\rho_{1} \int_{\Omega} u \psi_{1} & =\int_{\Omega \backslash A}\left[f(u)-f^{\prime}(u) u\right] \psi_{1}+\int_{A}\left[f(u)-f^{\prime}(u) u\right] \psi_{1} \\
& \geqq B_{1} \int_{\Omega \backslash A} \psi_{1}-O\left(1 /\|u\|_{\infty}\right) \\
& =B_{1} \int_{\Omega} \psi_{1}-\int_{A} \psi_{1}-O\left(1 /\|u\|_{\infty}\right) \\
& \geqq B_{1}-O\left(1 /\|u\|_{\infty}\right)
\end{aligned}
$$

and hence $\rho_{1}>0$ for $\|u\|_{\infty}$ large enough. Thus follows the stability of large positive solutions. The uniqueness of large positive solutions for $\lambda$ near $\mu_{1} / f^{\prime}(\infty)$ follows from an argument similar to that in Lemma 3.3.

\section{The radial case}

When $\Omega$ is a ball, positive solutions to (1.1)-(1.2) are known to be radially symmetric. Without loss of generality we may assume $\Omega$ to be the unit ball centered at the origin. Thus it suffices to study the equation

$$
\begin{gathered}
u^{\prime \prime}+((N-1) / r) u^{\prime}+\lambda f(u)=0 \text { for } r \in[0,1], \\
u^{\prime}(0)=0, \\
u(1)=0,
\end{gathered}
$$

where' denotes the differentiation with respect to $r=\|x\|$. For $d>0$, we define $u(\cdot, \lambda, d)$ to be the solution to $(4.1),(4.2)$ with $u(0, \lambda, d)=d$. We shall frequently write $u$ rather than $u(\cdot \lambda, d)$. It is well known and can be easily shown that if $u$ is a positive solution to (4.1)-(4.3), then $u(0)>\theta$. Let $S=\{(\lambda, u) \in \mathbb{R} \times \mathscr{C}(\bar{\Omega}):(\lambda, u)$ satisfies (4.1) (4.3)\}. We note that studying the behaviour of $S$ is equivalent to studying $\{(\lambda, d): u(1, \lambda, d)=0\}$. This follows from the continuous dependence of solutions to (4.1)-(4.3) on the initial conditions. We identify $S$ with the latter subset of $\mathbb{R}^{2}$. Using a rescaling (see [4]) and the uniqueness of the solution to the initial value problem (4.1) (4.2) when $u(0)=d$, we obtain

$$
u(r \rho, \lambda, d)=u\left(r, \lambda \rho^{2}, d\right) .
$$


Notation. Let $\hat{\mu}_{i}$ denote the eigenvalues of the problem:

$$
\begin{gathered}
\varphi^{\prime \prime}+((N-1) / r) \phi^{\prime}+\hat{\mu} \varphi=0 \quad \text { in }(0,1), \\
\varphi^{\prime}(0)=0, \quad \varphi(1)=0 .
\end{gathered}
$$

Using a comparison argument and the rescaling in (4.4), we obtain the following nonexistence result.

LEMMA 4.1. If $f$ satisfies (1.6), then (4.1)-(4.3) does not have positive solutions for $\lambda<\hat{\mu}_{1} / f^{\prime}(0)$ and for $\lambda>\hat{\mu}_{2} / f^{\prime}(\infty)$.

Proof. Let $(\lambda, d)$ be such that $u(\cdot, \lambda, d)$ is a positive solution to (4.1)-(4.3). Let $\varphi_{1}>0$ be an eigenfunction to (4.5)-(4.6) corresponding to the smallest eigenvalue $\hat{\mu}_{1}$. Now multiplying (1.1) by $\varphi_{1}$ and integrating, we obtain

$$
\int_{\Omega} \hat{\mu}_{1} \varphi_{1} u=\int_{\Omega}-\Delta \varphi_{1} u=\int_{\Omega} \lambda f(u) \varphi_{1} \leqq \int_{\Omega} \lambda f^{\prime}(0) \varphi_{1} u
$$

from (4.5) and using the fact that $f(t) \leqq f^{\prime}(0) t$ for all $t \geqq 0$. The above inequality is impossible if $\lambda \leqq \hat{\mu}_{1} / f^{\prime}(0)$ and hence $\lambda$ is bounded away from zero. Now, to prove the nonexistence of positive solutions for large $\lambda$, we proceed as follows. We extend $f$ to the left of 0 in such a way that $f^{\prime \prime}(t) \leqq 0$ for all $t \in \mathbb{R}$. Let $(\lambda, d)$ be such that $u(\cdot, \lambda, d)$ is a positive solution to (4.1)-(4.3). Using (4.4), we can choose $\zeta>\lambda$ such that $u(1, \zeta, d)=\beta$ and $u(\cdot, \zeta, d)$ has exactly two zeros in $(0,1)$. Thus $v(r):=u(\cdot, \zeta, d)-\beta$ satisfies

$$
\begin{gathered}
v^{\prime \prime}+((N-1) / r) v^{\prime}+\zeta\left(\frac{f(u)-f(\beta)}{u-\beta}\right) v=0, \\
v^{\prime}(0)=0, \quad v(1)=0,
\end{gathered}
$$

and $v$ has exactly one zero in $(0,1)$. Comparing this with $(4.5)$ for $\hat{\mu}=\hat{\mu}_{2}$, by Sturmian theory we conclude that there exists an $r_{0} \in(0,1)$ such that

$$
\zeta\left(\frac{f\left(u\left(r_{0}\right)\right)-f(\beta)}{u\left(r_{0}\right)-\beta}\right)<\hat{\mu}_{2} .
$$

Since

$$
\frac{f\left(u\left(r_{0}\right)\right)-f(\beta)}{u\left(r_{0}\right)-\beta}=f^{\prime}(a)
$$

for some $a, f^{\prime}(a)$ is bounded below by $f^{\prime}(\infty)$ (by using the concavity of $f$ ). This with (4.7) gives that

$$
\lambda<\zeta<\hat{\mu}_{2} / f^{\prime}(\infty)
$$

and hence the lemma is proved.

LeMmA 4.2. Let $\left(\lambda_{0}, d_{0}\right)$ be such that $u_{0}:=u\left(\cdot, \hat{\lambda}_{0}, d_{0}\right)$ is a positive solution to $(4.1)-(4.3)$ satisfying $u_{0}^{\prime}(1)=0$. If $(\lambda, d)$ is such that $u(\cdot, \lambda, d)$ is a positive solution to (4.1)-(4.3), then $d>d_{0}$. 
Proof. Let $u\left(\cdot, \lambda_{1}, d_{1}\right)$ be a positive solution to (4.1)-(4.3). Defining $u_{i}(r)=$ $u\left(r / \sqrt{\lambda_{i}}, \lambda_{i}, d\right)$, from (4.5) we infer that

$$
\begin{gathered}
u_{i}^{\prime \prime}+((N-1) / r) u_{i}^{\prime}+f\left(u_{i}\right)=0, \\
u_{i}^{\prime}(0)=0, \quad u_{i}\left(\sqrt{\lambda_{i}}\right)=0,
\end{gathered}
$$

for $i=0,1$. Let $d_{0}>d_{1}$. We first prove that $u_{0}$ and $u_{1}$ cannot meet above the $\beta$-level. For, let $u_{0}(r)>u_{1}(r)$ for $r \in[0, \bar{r})$ and let $u_{0}(\bar{r})=u_{1}(\bar{r})=a>\beta$. Thus $u_{0}^{\prime}(\bar{r})<u_{1}^{\prime}(\bar{r})<0$. Since $u_{0}(r)>u_{1}(r)>\beta$ for $r \in[0, \bar{r})$, the concavity of $f$ gives

$$
\left(u_{1}(r)-\beta\right) f\left(u_{0}(r)\right) \leqq\left(u_{0}(r)-\beta\right) f\left(u_{1}(r)\right) \text { on }[0, \bar{r}) ;
$$

that is,

$$
\left(u_{1}(r)-\beta\right)\left(r^{N-1} u_{0}^{\prime}\right)^{\prime} \geqq\left(u_{0}(r)-\beta\right)\left(r^{N-1} u_{1}^{\prime}\right)^{\prime} \quad \text { on }[0, \bar{r}) ;
$$

that is,

$$
\left[\left(u_{1}(r)-\beta\right) r^{N-1} u_{0}^{\prime}\right]^{\prime} \geqq\left[\left(u_{0}(r)-\beta\right) r^{N-1} u_{1}^{\prime}\right]^{\prime} \quad \text { on }[0, \bar{r}) \text {. }
$$

Integrating this over $(0, \bar{r})$, we get $u_{0}^{\prime}(\bar{r}) \geqq u_{1}^{\prime}(\bar{r})$, which is a contradiction. Now, let $u_{1}(r)>u_{0}(r)$ for $r \in\left(\bar{r}, \sqrt{\min \left\{\lambda_{0}, \lambda_{1}\right\}}\right)$ and $u_{0}(\bar{r})=u_{1}(\bar{r})=a \leqq \beta$. Thus $u_{0}^{\prime}(\bar{r})<u_{1}^{\prime}(\bar{r})<0$. Multiplying $(4.8)$ by $r^{2 N-2} u_{i}^{\prime}$ and integrating over $\left(\bar{r}, \sqrt{\lambda_{i}}\right)$, we obtain

$$
{\sqrt{\lambda_{i}}}^{2 N-2}\left(u_{i}^{\prime}\left(\sqrt{\lambda_{i}}\right)\right)^{2}-\bar{r}^{2 N-2}\left(u_{i}^{\prime}(\bar{r})\right)^{2}=2 \int_{0}^{a} r_{i}^{2 N-2}(u) f(u) d u,
$$

where $r_{i}(u)$ represents the inverse function to $u_{i}$ (i.e. $r_{i}:\left[0, d_{i}\right] \rightarrow\left[0, \sqrt{\lambda_{i}}\right]$ with $u_{i}\left(r_{i}(u)\right)=u$ for $\left.0 \leqq u \leqq d_{i}, i=0,1\right)$. This, in turn, implies that

$$
\begin{gathered}
{\sqrt{\lambda_{1}}}^{2 N-2}\left(u_{1}^{\prime}\left(\sqrt{\lambda_{1}}\right)\right)^{2}+\bar{r}^{2 N-2}\left[\left(u_{0}^{\prime}(\bar{r})\right)^{2}-\left(u_{1}^{\prime}(\bar{r})\right)^{2}\right] \\
=2 \int_{0}^{a}\left[r_{1}^{2 N-2}(u)-r_{0}^{2 N-2}(u)\right] f(u) d u .
\end{gathered}
$$

(Note that here we have used $u_{0}^{\prime}\left(\sqrt{\lambda_{0}}\right)=0$.) This is a contradiction, since the left side of the above equation is positive and the right side is negative. Thus we conclude that $d_{1}>d_{0}$ and hence the lemma is proved.

LEMMA 4.3. If $f$ satisfies (1.6), then (4.1)-(4.3) has at most one positive solution with $u^{\prime}(1, \lambda, d)=0$.

Proof. Suppose on the contrary that $u\left(\cdot, \lambda_{i}, d_{i}\right)$ is a positive solution to (4.1)-(4.3) satisfying $u^{\prime}\left(1, \lambda_{i}, d_{i}\right)=0$ for $i=0,1$. Then by Lemma 4.2 we get $d_{0}<d_{1}$ and $d_{1}<d_{0}$, which are contradictory. Hence the lemma is proved.

We denote the derivatives of $u$ with respect to $\lambda$ and $d$ by $u_{\lambda}$ and $u_{d}$, respectively. Differentiation of (4.4) with respect to $\rho$ results in

$$
u_{\lambda}(r, \lambda, d)=r u^{\prime}(r, \lambda, d) / 2 \lambda .
$$

Differentiating (4.1) with respect to $d$, we see that $u_{d}$ satisfies the corresponding linearised problem:

$$
\begin{gathered}
u_{d}^{\prime \prime}+((N-1) / r) u_{d}^{\prime}+\lambda f^{\prime}(u) u_{d}=0, \\
u_{d}(0)=1, \quad u_{d}^{\prime}(0)=0 .
\end{gathered}
$$


The following result on the zeros of $u_{d}$ is from [3]. We include it here for the sake of completeness.

LEMMA 4.4. If $u$ is a positive solution to (4.1)-(4.3), then $u_{d}$ has at most one zero in $[0,1]$.

REMARK 4.5. Lemmas 4.1 and 4.2 hold for any monotonically increasing semipositone concave nonlinearity $f$. Also, note that Lemma 4.2 establishes that if $\lambda_{0}<\lambda_{1}$, then $u_{0}(r)<u_{1}(r)$ in $\left[0, \sqrt{\lambda_{0}}\right]$.

\section{Proofs of theorems}

Proof of Theorem 1.2. The nonexistence of positive solutions to (1.1)-(1.2) follows from Lemma 4.1. The stability and the uniqueness results follow from Lemmas 3.2, 3.3 and 3.4. If $\left\{\lambda_{j}\right\}$ is bounded and $\left\{\left(\lambda_{j}, u_{j}\right)\right\}$ satisfies (1.1)-(1.2) with $u_{j}>0$ in $\Omega$ and $\left\|u_{j}\right\|_{\infty} \rightarrow \infty$, then $\left\{u_{j} /\left\|u_{j}\right\|_{\infty}\right\}$ converges to a function $v>0$ that satisfies $-\Delta v=$ $\hat{\lambda} f^{\prime}(\infty) v$, where $\hat{\lambda}$ is an accumulation point of $\left\{\lambda_{j}\right\}$. Since $\hat{\lambda} f^{\prime}(\infty)=\mu_{1}, \hat{\lambda}$ is the only accumulation point of $\left\{\lambda_{j}\right\}$, and hence there follows the uniqueness of large positive solutions for $\lambda$ 's in bounded intervals.

Proof of Theorem 1.3. If (1.4) holds, then from Lemmas 3.2 and 3.3 we obtain that for $\lambda$ sufficiently close to $\mu_{1} / f^{\prime}(\infty)$ there exists a unique unstable positive solution. In fact, there exist $c_{1}, \varepsilon_{1}>0$ and a continuous decreasing function $\sigma:\left[c_{1},+\infty\right) \rightarrow\left[\mu_{1} / f^{\prime}(\infty), \infty\right)$ such that $\lim _{d \rightarrow \infty} \sigma(d)=\mu_{1} / f^{\prime}(\infty)$ and if $\|u\|_{\infty} \geqq c_{1}$ and $\left|\lambda-\mu_{1} / f^{\prime}(\infty)\right|<\varepsilon_{1}$, then $u=u\left(\cdot, \sigma\left(\|u\|_{\infty}\right),\|u\|_{\infty}\right)$. Let $\Gamma \subset S$ denote the connected component of solutions to (4.1)-(4.3) containing $\left\{(\sigma(d), d): d \in\left[c_{1},+\infty\right)\right\}$. Let $d_{2} \equiv \inf \{c: \sigma$ can be extended as a continuous function from $[c,+\infty) \rightarrow\left(\mu_{1} / f^{\prime}(\infty), \infty\right)$ such that $\left.(\sigma(d), d) \subset \Gamma\right\}$. Note that $d_{2} \geqq \theta$. We define $\lambda_{2} \equiv \sup \left\{\sigma(d): d>d_{2}\right\}$. By Lemma 4.1, $\lambda_{2}<\infty$. Also, $u_{\lambda}\left(1, \lambda_{2}, d_{2}\right)=0$. For, otherwise, from (4.10) we have $u_{\lambda}\left(1, \lambda_{2}, d_{2}\right)<0$, which implies that $\sigma$ can be extended to the left of $d_{2}$, a contradiction to the definition of $d_{2}$. Now, using Lemmas 3.3 and 3.4, we have $u_{d}(1)<0$ and hence $\sigma^{\prime}(d)<0$. Thus, for any $\lambda \in\left[\mu_{1} / f^{\prime}(\infty), \lambda_{2}\right]$, there exists a unique $d$ such that $(\lambda, d) \in \Gamma$. From the uniqueness of degenerate positive solution (see Lemma 4.3) we conclude that $\Gamma \equiv S$. This compltes the proof of (i).

If (1.5) holds, then from Lemmas 3.2 and 3.4 we obtain that for $\lambda$ sufficiently close to $\mu_{1} / f^{\prime}(\infty)$ there exists a unique stable positive solution. In fact, there exist $c_{1}, \varepsilon_{1}>0$ and a continuous increasing function $\sigma:\left[c_{1},+\infty\right) \rightarrow\left[\mu_{1} / f^{\prime}(\infty), \infty\right)$ such that $\lim _{d \rightarrow \infty} \sigma(d)=\mu_{1} / f^{\prime}(\infty)$ and if $\|u\|_{\infty} \geqq c_{1}$ and $\left|\lambda-\mu_{1} / f^{\prime}(\infty)\right|<\varepsilon_{1}$ then $u=$ $u\left(\cdot, \sigma\left(\|u\|_{\infty}\right),\|u\|_{\infty}\right)$. Let $\Gamma \subset S$ denote the connected component of solutions to (4.1)-(4.3) containing $\left\{(\sigma(d), d): d \in\left[c_{1},+\infty\right)\right\}$. Let $d_{1} \equiv \inf \{c: \sigma$ can be extended as a continuous function from $[c,+\infty) \rightarrow\left(\mu_{1} / f^{\prime}(\infty), \infty\right)$ such that $\left.(\sigma(d), d) \subset \Gamma\right\}$. Note that $d_{1} \geqq \theta$. We define $\lambda_{1} \equiv \inf \left\{\sigma(d): d>d_{1}\right\}$. By Lemma 4.1, $\lambda_{1}>0$. Also, $u_{d}\left(1, \lambda_{1}, d_{1}\right)=0$, for otherwise $u_{d}\left(1, \lambda_{1}, d_{1}\right)>0$ which implies that $\sigma$ can be extended to $\left[d_{1}-\varepsilon, \infty\right)$, which contradicts the definition of $d_{1}$. By $[3$, Lemma 2], $u_{\lambda}\left(1, \lambda_{1}, d_{1}\right)<0$ and $u_{d d}\left(1, \lambda_{1}, d_{1}\right)>0$. These imply that there is a differentiable function $\Lambda:\left(d_{1}-\varepsilon, d_{1}+\varepsilon\right) \rightarrow \mathbb{R}$ such that $u(\cdot, \Lambda(d), d)$ is a solution to (4.1)-(4.2) for any $d \in\left(d_{1}-\varepsilon, d_{1}+\varepsilon\right)$. In addition, $\Lambda^{\prime}\left(d_{1}\right)=0$ with $\Lambda^{\prime \prime}\left(d_{1}\right)>0$. We define $d_{2}=\inf \{c: \Lambda$ can be extended as a continuous function from $\left[c, d_{1}\right) \rightarrow \mathbb{R}$ with $\left.(\Lambda(d), d) \subset \Gamma\right\}$. Note that $d_{2} \geqq \theta$. We define $\lambda_{2} \equiv \sup \left\{\Lambda(d): d>d_{2}\right\}$. By Lemma 4.1, $\lambda_{2}<\infty$. Also, 
A. Castro et al.

$u_{\lambda}\left(1, \lambda_{2}, d_{2}\right)=0$. For, otherwise, from (4.10) we have $u_{\lambda}\left(1, \lambda_{2}, d_{2}\right)<0$, which implies that $\Lambda$ can be extended to the left of $d_{2}$, a contradiction to the definition of $d_{2}$. Now, using Lemmas 3.4 and 4.4 , we have $u_{d}(1)<0$ and hence $\Lambda^{\prime}(d)<0$. From the uniqueness of degenerate positive solution (see Lemma 4.3) we conclude that $\Gamma \equiv S$. This completes the proof of (ii).

\section{References}

1 R. Adams. Sobolev Spaces (New York: Academic Press, 1975).

2 A. Ambrosetti, D. Arcoya and B. Buffoni. Positive solutions for some semipositone problems via Bifurcation theory. Differential Integral Equations 7 (1994), 655-64.

3 A. Castro and S. Gadam. Uniqueness of stable and unstable positive solutions for semipositone problems. Nonlinear Anal. 22 (1994), 425-9.

4 A. Castro, S. Gadam and R. Shivaji. Branches of radial solutions for semipositone problems. J. Differential Equations 120 (1995), 30-45.

5 A. Castro, S. Gadam and R. Shivaji. Evolution of positive solution curves in semipositone problems with concave nonlinearities (Preprint).

6 A. Castro, J. Garner and R. Shivaji. Existence results for classes of sublinear semipositone problems. Results Math. 23 (1993), 214-20.

7 A. Castro and R. Shivaji. Positive solutions for a concave semipositone Dirichlet problem. Nonlinear Anal. (to appear).

8 M. G. Crandall and P. H. Rabinowitz. Bifurcation from simple eigenvalues. J. Funct. Anal. 8 (1971), 321-40.

9 S.-H. Wang. Positive solutions for a class of nonpositone problems with concave nonlinearities. Proc. Roy. Soc. Edinburgh Sect. A 124 (1994), 507-15. 